Agrotrópica 32(3): 189 - 196. 2020.

Centro de Pesquisas do Cacau, Ilhéus, Bahia, Brasil

\title{
FUNGOS MICORRÍZICOS ARBUSCULARES NA CULTURA DO CACAUEIRO I: OCORRÊNCIA E DIVERSIDADE EM SISTEMAS CACAU-CABRUCA
}

\author{
Maria Aparecida N. Santos ${ }^{1}$, Carla S. Sousa ${ }^{2}$ Eduardo Gross ${ }^{3}$, Leoberto J. X. Oliveira Filho \\ Thâmara M. Lima ${ }^{3}$, Bruno T. Goto ${ }^{4}$
}

\begin{abstract}
${ }^{1}$ Universidade Federal do Recôncavo da Bahia. Rua G, 99A, Vereador Acácio Almeida, Itajuípe, Ba, Brasil. aparecida.santos13@bol.com.br. ${ }^{2}$ Instituto Federal de Educação, Ciência e Tecnologia Baiano-Campus Alagoinhas. Rua Manoel Romão, 150, Alagoinhas Velha - Alagoinhas, Ba, Brasil. cssagro@yahoo.com.br. ${ }^{3}$ Universidade Estadual de Santa Cruz-Campus Soane Nazaré de Andrade, Rodovia Ilhéus/Itabuna, km 16, Bairro Salobrinho, Ilhéus, Ba, Brasil. egross@uesc.br; 1leo.agro@gmail.com; thamaralima6@gmail.com..Universidade Federal do Rio Grande do Norte. Avenida Senador Salgado Filho, 3000, Lagoa nova, Natal, RN, Brasil.brunogoto@hotmail.com.
\end{abstract}

O presente estudo teve como objetivo avaliar a ocorrência e diversidade de fungos micorrízicos arbusculares (FMAs) em plantios de cacau-cabruca localizados na região do Sul da Bahia. Inicialmente, foram realizadas coletas de amostras de solo e raízes de plantas de cacaueiro, em dois períodos climáticos (seco e chuvoso), em seis áreas de cacau-cabruca. Nas amostras, foram realizadas: avaliação da densidade de esporos, caracterização química do solo, identificação taxonômica das espécies e avaliação da taxa de colonização micorrízica das raízes. Com exceção da área 1, que apresentou maior densidade de esporos no período chuvoso, a maior densidade de esporos foi registrada nas amostras de solo coletadas no período seco. Não houve interferência do período de coleta sobre a taxa de colonização das raízes das plantas de cacau. Foram identificadas um total de 26 espécies de fungos micorrízicos arbusculares, sendo registrada maior riqueza de espécies nas áreas 2 (11 espécies), com predominância dos gêneros Glomus e Acaulospora. Houve correlação positiva significativa entre taxa de colonização das raízes e os teores de $\mathrm{Mg}$ e $\mathrm{pH}$ do solo.

Palavras-chave: micorrização, Theobroma cacao L., identificação taxonômica.

Arbuscular mycorrhizal fungi in the cacaueiro I culture: Occurrence and diversity in cocoa-cabruca systems. The present study aimed to evaluate the occurrence and diversity of arbuscular mycorrhizal fungi (AMFs) in cocoa-cabruca plantations located in the southern region of Bahia. Initially, samples were collected from soil and roots of cacao plants, in two climatic periods (dry and rainy), in six areas of cacao-cabruca. In the samples, were carried out: evaluation of spore density, chemical characterization of the soil, taxonomic identification of the species and evaluation of the rate of mycorrhizal colonization of the roots. With the exception of area 1, which showed the highest spore density in the rainy season, the highest spore density was recorded in soil samples collected in the dry period. There was no interference from the collection period on the rate of colonization of the roots of cocoa plants. A total of 26 species of arbuscular mycorrhizal fungi were identified, with greater species richness recorded in areas 2 (11 species), with predominance of the genera Glomus and Acaulospora. There was a significant positive correlation between root colonization rate and soil $\mathrm{Mg}$ and $\mathrm{pH}$ levels.

Key words: Mycorrhization, Theobroma cacao L., taxonomic identification. 


\section{Introdução}

O cacaueiro (Theobroma cacao L.), pertencente à família Malvaceae, é uma planta perenefólia, originária da América do Sul. Considerada uma das culturas de grande importância econômica, o cacau é mundialmente comercializado, sobretudo, pelo valor de suas sementes, matéria prima na fabricação de chocolate e na utilização da manteiga para fabricação de fármacos e cosméticos (Vásquez et al., 2019).

O cultivo do cacau na região Sul da Bahia é realizado tradicionalmente sob o sistema sombreado (cabruca), esta forma de plantio tem sido importante para conservação da comunidade animal e vegetal da Mata Atlântica na região (Vanhove, Vanhoudt \& Van Damme, 2016; Toledo-Hernández, Wanger \& Tscharntke, 2017). Entretanto, houve redução nos preços do produto no mercado externo, resultante da grande crise cacaueira proveniente da devastação das lavouras causada pela "vassoura de bruxa", uma das principais doenças que acomete o cacaueiro, causada pelo fungo Moniliophthora perniciosa (Stahel) Aime $\&$ Phillips-Mora (Benjamin et al., 2016).

Estudos revelam que a comunidade microbiana do solo através de seus processos biológicos desempenha papel fundamental na manutenção de diversos agroecossistemas e ecossistemas naturais (Prado et al., 2019). Dentre os participantes desses processos, os fungos micorrízicos arbusculares (FMAs) ganham destaque, por estabelecerem simbiose mutualística com a maioria das plantas (Dodd, 2000).

A diversidade de espécies de fungos micorrízicos arbusculares presente em ecossistemas naturais e agroecossistemas desempenha funções importantes na estruturação e manutenção da microbiota dos solos, mantém a produtividade da comunidade vegetal, além de atuar na estruturação e estabilidade dos agregados do solo (Wang, 2017; Yang et al., 2016; Prado et al., 2019). A simbiose entre FMAs e as plantas hospedeiras oferece grandes benefícios para estas, pois promove a ampliação da área de absorção de água e nutrientes pelas hifas, proporcionando melhor desenvolvimento e produtividade de diversas culturas (Li et al., 2019; Aggangan, Cortes \& Reaño, 2019; Frew, 2019; Thioub et al., 2019), sendo o cacaueiro uma das espécies beneficiadas por esta associação.
Diante do exposto, o presente trabalho propôs realizar um levantamento da ocorrência e diversidade de espécies de fungos micorrízicos arbusculares em cacaueiros cultivados sob sistema cabruca, visando conhecer sobre a simbiose entre estes fungos e a cultura e permitir, a partir de novos estudos utilizar esses microrganismos como tecnologia para promover maiores rendimentos na produção cacaueira, e proporcionar melhor custo benefício nos sistemas produtivos.

\section{Material e Métodos}

\section{Caracterização das áreas de coleta}

As coletas foram realizadas em seis propriedades localizadas na região Sul da Bahia, situadas entre os municípios de Itajuípe e Ilhéus. As propriedades apresentavam algumas características semelhantes entre si: pertencentes a agricultores familiares, com até quatro módulos fiscais, ( $\leq 80$ hectares); plantios de cacau com idade entre cinco a dez anos de implantação, com pouco ou nenhum manejo. As variedades de cacau predominante nessas áreas foram: CCN51, CEPEC 2002, PH 16 e SJ 02, clones mais utilizados pelos cacauicultores da região por apresentar alta produtividade; maior resistência às doenças vassoura de bruxa e podridão parda; e autocompatibilidade.

As áreas estão localizadas nas seguintes coordenadas geográficas: Área 1-14 ${ }^{\circ} 37^{\prime} 55.2^{\prime \prime S}$ $39^{\circ} 23^{\prime} 56.2^{\prime} \mathrm{W}$; Área 2 - $14^{\circ} 37^{\prime} 45.2^{\prime} \mathrm{S} 39^{\circ} 24^{\prime} 10.5^{\prime} \mathrm{W}$; Área 3 - $14^{\circ} 37^{\prime} 34.5^{\prime} \mathrm{S} 39^{\circ} 24^{\prime} 23.1^{\prime} \mathrm{W}$; Área 4 -

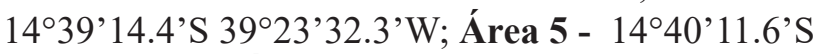
$39^{\circ} 23^{\prime} 37.1^{\prime} \mathrm{W}$; Área 6 - $14^{\circ} 41^{\prime} 00.4^{\prime} \mathrm{S} 39^{\circ} 24^{\prime} 22.4^{\prime} \mathrm{W}$; com topografia plana, solos bem drenados, propício ao cultivo do cacaueiro.

A região apresenta alta pluviosidade ao longo do ano, mesmo em períodos secos, chegando a $1.495 \mathrm{~mm}$ de pluviosidade média anual nas áreas localizadas no município de Itajuípe e 1.946 mm nas áreas localizadas no município de Ilhéus.

\section{Coleta das amostras de solo e raízes de plantas de cacau}

Em cada área, foram selecionadas 5 plantas de cacaueiro, e coletadas amostras de solo rizosférico na camada $0-15 \mathrm{~cm}$ de profundidade, em dois períodos 
climáticos chuvoso (junho/2018) e seco (março/2019). As coletas foram realizadas no mesmo dia em todas as áreas. As amostras de solo após coleta, foram secas ao ar, destorroadas, homogeneizadas, peneiradas em peneira de $2 \mathrm{~mm}$, e em seguida armazenadas a $4^{\circ} \mathrm{C}$ em câmara fria, para conservação. Foram separadas das amostras, raízes finas de cacaueiro $(<2 \mathrm{~mm})$, que foram lavadas em água e acondicionadas em recipientes plásticos, previamente identificados, contendo álcool $50 \%$, para conservação.

Para realização da caracterização química (Tabela 1), as amostras de solo rizosférico coletadas em cada área foram misturadas (formando uma amostra composta/área), secas ao ar, destorroadas, homogeneizadas, peneiradas em peneira de $2 \mathrm{~mm}$ e analisadas no Laboratório de Química do Solo, do Departamento de Ciência do Solo, da Escola Superior de Agricultura "Luiz de Queiroz" (Esalq-USP).

\section{Determinação da densidade de esporos}

Os esporos de FMA foram extraídos de $50 \mathrm{~g}$ das amostras de solo (Gerdemann \& Nicolson, 1963), por meio da metodologia do peneiramento úmido, seguido por centrifugação em água e solução de sacarose 50\% (Jenkins, 1964). Neste procedimento, cada amostra de solo foi transferida para um recipiente contendo $1000 \mathrm{~mL}$ de água, homogeneizados com a mão para desestruturação de todos os torrões (aglomerados) existentes.

$\mathrm{Na}$ sequência, o material foi decantado por um minuto, sendo o sobrenadante vertido sobre duas peneiras sobrepostas de 40 e 400 mesh. O material retido nas peneiras foi recolhido em um tubo de ensaio e submetido à centrifugação em água (3000 rpm) por 3 minutos. Em seguida o sobrenadante foi descartado e, ao material depositado no fundo, adicionou-se solução de sacarose $50 \%$. O material foi ressuspenso com auxílio de bastão de vidro e os tubos de ensaio novamente levados à centrífuga $(2000 \mathrm{rpm})$ por 1 minuto.

O sobrenadante foi vertido na peneira de 400 mesh e os esporos retidos na mesma foram lavados em água corrente para retirada do excesso de sacarose, e transferidos para placa canaletada, para serem quantificados com auxílio de um microscópio estereoscópico $(40 \mathrm{x})$.

\section{Determinação da taxa de colonização micorrízica}

Para determinação da taxa de colonização micorrízica, as amostras de raízes foram lavadas repetidas vezes em água destilada e em seguida imersas em solução de $\mathrm{NaOH} 10 \%$ por $24 \mathrm{~h}$, em temperatura ambiente, para serem diafanizadas. Após esse período, foram lavadas com água destilada, tratadas com $\mathrm{H}_{2} \mathrm{O}_{2}$ alcalina por 45 minutos e em seguida com $\mathrm{HCl} 1 \%$ por 3 minutos. Posteriormente, foram imersas em solução de azul de trypan $0,05 \%$ lactoglicerol por $24 \mathrm{~h}$, em temperatura ambiente (Koske \& Gemma, 1989), e após este período, conservadas em lactoglicerol ácido, até avaliação da percentagem de colonização micorrízica, a qual foi realizada pelo método da placa quadriculada (Giovannetti \& Mosse, 1980).

Os fragmentos de raízes coradas foram colocados em placa quadriculada (quadrículas de 1,27 cm) e observados em microscópio estereoscópico (40x), sendo contados 100 segmentos de raiz que fizeram interseção com as linhas verticais e horizontais e registrando-se o número de segmentos colonizados. Foram considerados colonizados, os segmentos de

Tabela 1. Análise química do solo nas áreas de cacau cabruca na região Sul da Bahia, 2018

\begin{tabular}{|c|c|c|c|c|c|c|c|}
\hline Determinações & Unidade & Área 1 & Área 2 & Área 3 & Área 4 & Área 5 & Área 6 \\
\hline $\mathrm{pH} \mathrm{CaCl}_{2}$ & - & 4,7 & 5,0 & 4,9 & 4,6 & 4,6 & 4,9 \\
\hline M.O. colorimétrico & g.dm.3 & 35 & 32 & 21 & 33 & 35 & 31 \\
\hline P Resina & $\mathrm{mg} \cdot \mathrm{dm}^{\cdot 3}$ & 18 & 14 & 12 & 11 & 17 & 9 \\
\hline K Resina & mmolc. $\mathrm{dm}^{\cdot 3}$ & 1,2 & $<0,9$ & 1,6 & 2 & 2,4 & 1,9 \\
\hline Ca Resina & mmolc. $\mathrm{dm}^{\cdot 3}$ & 29 & 28 & 20 & 25 & 28 & 28 \\
\hline Mg Resina & mmolc. $\mathrm{dm}^{\cdot 3}$ & 16 & 22 & 14 & 9 & 11 & 14 \\
\hline $\mathrm{H}+\mathrm{Al} \mathrm{SMP}$ & mmolc. $\mathrm{dm}^{\cdot 3}$ & 58 & 34 & 42 & 52 & 52 & 28 \\
\hline $\mathrm{SB}$ & mmolc. $\mathrm{dm}^{\cdot 3}$ & 46,2 & 50,8 & 35,6 & 36 & 41,4 & 43,9 \\
\hline СTC & mmolc.dm. ${ }^{3}$ & 104,2 & 84,8 & 77,6 & 88 & 93,4 & 71,9 \\
\hline $\mathrm{V}$ & $\%$ & 44 & 60 & 46 & 41 & 44 & 61 \\
\hline
\end{tabular}


raízes que apresentaram estruturas típicas de fungos micorrízicos, tais como vesículas, arbúsculos, hifas e pelotões.

\section{Identificação taxonômica das espécies de FMAs}

Para identificação taxonômica das espécies de FMA, foram montadas lâminas com PVLG (álcool polivinílico-lactoglicerol) e com Melzer + PVLG (1:1; v:v) (Morton, Bentivenga \& Wheeler, 1993), a partir das extrações realizadas para contagem de esporos. Esses esporos foram agrupados de acordo com as características morfológicas (cor, tamanho, forma) e dispostos nas lâminas para identificação. Para subsidiar a identificação, utilizou-se literatura especializada (Schenck \& Perez, 1988; Carrenho, 1998). A riqueza de espécies foi determinada de acordo com o número de espécies de FMAs que ocorreu em cada área.

\section{Delineamento experimental e análise estatística}

$\mathrm{O}$ experimento foi conduzido em delineamento inteiramente casualizado, esquema fatorial $6 \times 2$, sendo 6 áreas e 2 períodos de coleta (chuvoso e seco), com cinco repetições.

Os dados referentes a densidade de esporos e a colonização micorrízica foram transformados, respectivamente, por $(x+0,5)^{1 / 2}$ e $\operatorname{arc} \operatorname{sen} \sqrt{x / 100}$. Foram realizadas análises de correlação simples de Pearson (r) entre a densidade de esporos, colonização micorrízica e as características químicas do solo. A significância do coeficiente de correlação (r) foi verificada pelo teste t de Student, a $5 \%$ de probabilidade. Todas as análises estatísticas foram realizadas utilizandose o programa R (R Core Team, 2019).

\section{Resultados e Discussão}

Houve efeito significativo da interação entre os fatores período e área de coleta das amostras apenas sobre a densidade de esporos $(\leq 0,001)$ (Tabela 2$)$. A variável taxa de colonização micorrízica das raízes pelos fungos micorrízicos arbusculares foi influenciada apenas pelo fator área de coleta $(\leq 0,001)$. A densidade de esporos dos fungos micorrízicos arbusculares teve efeito significativo dos fatores isolados área e período de coleta das amostras.
Tabela 2. Resumo da Análise de variância da densidade de esporos (DE) e taxa de colonização micorrízica (CM) de raízes de cacaueiro por fungos micorrízicos arbusculares em plantios de cacau cabruca na região Sul da Bahia, 2018

\begin{tabular}{llc}
\hline \multirow{2}{*}{ Fonte de variação } & \multicolumn{2}{c}{ QM } \\
\cline { 2 - 3 } & \multicolumn{1}{c}{$\mathbf{D E}$} & $\mathbf{C M}$ \\
\hline Área de coleta & $0,25760^{* * *}$ & $799,07^{* * *}$ \\
Período de coleta & $0,62467^{* * *}$ & 55,01 \\
Área x Período & $0,24565^{* * *}$ & 74,99 \\
Resíduo & 0,02683 & 123,08 \\
$\mathrm{CV} \%$ & 29,56 & 20,0 \\
\hline \multicolumn{2}{c}{$* * * \mathrm{e}^{*}$ significativo $(\mathrm{p} \leq 0,001)$ e $(\mathrm{p} \leq 0,05)$, respectivamente, } \\
pelo teste F. QM = quadrado médio; CV\% = coeficiente de variação.
\end{tabular}

A densidade de esporos nas áreas avaliadas variou entre 72,4 a 562,8 esporos por $50 \mathrm{~g}$ de solo durante o período chuvoso, enquanto que no período seco foram registrados valores entre 90,8 a 671,8 esporos por 50 g de solo (Tabela 3).

$\mathrm{Na}$ coleta realizada durante o período chuvoso observou-se maior densidade de esporos na área 1 (562,8 esporos por $50 \mathrm{~g}$ de solo), diferindo estatisticamente dos valores registrados nas áreas 2 , $3,4,5$ e 6 , as quais não diferiram estatisticamente entre si, apresentando, respectivamente: 284,$0 ; 147,6 ; 91,2$; 138,2 e 72,4 esporos por $50 \mathrm{~g}$ de solo.

No período seco, a área 2 apresentou maior densidade de esporos (671,8 esporos por $50 \mathrm{~g}$ de solo), seguida da área 4 (475,6 esporos por $50 \mathrm{~g}$ de solo). As áreas 1, 3 e 6 não apresentaram diferença estatística entre si $(300,4 ; 349,2 ; 392,6$ esporos por $50 \mathrm{~g}$ de solo). $\mathrm{Na}$ área 5 , registrou-se o menor valor referente à

Tabela 3. Densidade de esporos (DE) de fungos micorrízicos arbusculares, durante dois períodos de coleta, em plantios de cacau cabruca na região Sul da Bahia, 2018

\begin{tabular}{lcc}
\hline \multirow{2}{*}{ Área } & \multicolumn{2}{c}{ DE (por 50 g de solo) } \\
\cline { 2 - 3 } & Período chuvoso & Período seco \\
\hline Área 1 & $562,8 \mathrm{aA}^{*}$ & $300,4 \mathrm{cB}$ \\
Área 2 & $284,0 \mathrm{bB}$ & $671,8 \mathrm{aA}$ \\
Área 3 & $147,6 \mathrm{bB}$ & $349,2 \mathrm{cA}$ \\
Área 4 & $91,2 \mathrm{bB}$ & $475,6 \mathrm{bA}$ \\
Área 5 & $138,2 \mathrm{bA}$ & $90,8 \mathrm{dA}$ \\
Área 6 & $72,4 \mathrm{bB}$ & $392,6 \mathrm{cA}$ \\
\hline
\end{tabular}

*Médias seguidas pelas mesmas letras não diferem estatisticamente entre si pelo teste de Scott-Knott a 5\% de significância. Letras maiúsculas comparam nas linhas cada área em ambos os períodos de coleta. Letras minúsculas comparam na coluna as áreas em cada período de coleta. 
densidade de esporos, em comparação com as demais áreas (90,8 esporos por $50 \mathrm{~g}$ de solo).

Em relação à densidade de esporos nas áreas entre os períodos de coleta, verificou-se que na área 1 , foram registrados maiores valores durante o período chuvoso em comparação ao período seco, e estes períodos diferiram estatisticamente entre si $(562,8$ e 300,4 esporos por $50 \mathrm{~g}$ de solo, respectivamente). As áreas 2, 3, 4 e 6 apresentaram maiores valores referentes à densidade de esporos no período seco $(349,2$ a 671,8 esporos por $50 \mathrm{~g}$ de solo) em relação ao período chuvoso (72,4 a 284,0 esporos por $50 \mathrm{~g}$ de solo). Não houve diferença significativa na densidade de esporos entre o período seco e chuvoso na área 5.

Observa-se que de um modo geral, houve maior esporulação dos fungos no período seco em comparação ao período chuvoso (Tabela 3). Segundo Bonfim et al. (2010) em condições ambientais adversas algumas espécies de FMAs desenvolvem estratégias de sobrevivência e manutenção da espécie através do aumento da esporulação.

A taxa de colonização micorrízica das raízes de cacaueiro variou entre $47 \%$ a $81 \%$ (Tabela 4 ). Estes valores são superiores aos observados por Gross e Pereira (2018) em plantas de cacaueiro que variaram entre 21 a 36\%. Oliveira Filho (2019), observou valores referentes a taxa de colonização micorrízica em raízes de cacaueiros variando entre $15,9 \%$ a $57,8 \%$ em sistemas cacau cabruca na região Sul da Bahia. Não houve influência do período de coleta das amostras sobre a taxa de colonização das raízes de cacaueiro, sendo observada apenas diferença com relação à área coletada.

Observa-se que as plantas de cacaueiro presentes na área 2 apresentaram maior taxa de colonização

Tabela 4. Taxa de colonização micorrízica (CM) das raízes de cacaueiro em plantios de cacau-cabruca na região Sul da Bahia, 2018

\begin{tabular}{cc}
\hline Área & CM (\%) \\
\hline Área 1 & $69 \mathrm{a}^{*}$ \\
Área 2 & $81 \mathrm{a}$ \\
Área 3 & $76 \mathrm{a}$ \\
Área 4 & $52 \mathrm{~b}$ \\
Área 5 & $47 \mathrm{~b}$ \\
Área 6 & $75 \mathrm{a}$
\end{tabular}

*Médias seguidas por letras iguais não diferem estatisticamente entre si pelo teste de Scott-Knott a 5\% de probabilidade. micorrízica nas raízes (81\%), contudo, não diferiram estatisticamente das plantas cultivadas nas áreas 1, 3 e $6(69 \%, 76 \%$ e $75 \%$, respectivamente). Menores valores referentes a taxa de colonização micorrízica foram registrados nas raízes das plantas de cacaueiro presentes nas áreas 4 e 5, e estas não diferiram estatisticamente entre si, apresentando $52 \%$ e $47 \%$, respectivamente. Segundo Lima e Sousa (2014), fatores edafoclimáticos e aspectos genéticos da relação fungo-planta influenciam na taxa de colonização e na resposta à micorrização.

Foram identificadas um total de 23 espécies de FMAs nas áreas de cacau-cabruca avaliadas, pertencentes aos gêneros Acaulospora (8), Ambispora (1), Claroideoglomus (1), Dominikia (1), Funneliformis (1), Gigaspora (1), Glomus (5), Paraglomus (2), Sclerocystis (1), Septoglomus (2) (Tabela 5). A área 3 apresentou maior riqueza de espécie (11), predominando os gêneros Glomus e Acaulospora. A segunda maior riqueza de espécie ocorreu na área 2 (10), seguida das áreas 1(9), 4(6) e 5 (6). Na área 6 foi registrada menor riqueza de espécie (4), composta apenas por espécies do gênero Acaulospora. Alguns fatores influenciam na ocorrência e diversidade de FMAs, tais como características edáficas, espécies de plantas, presença ou não de cobertura vegetal, condições ambientais e manejo do solo (Eom, Hartnett \& Wilson, 2000; Moreira e Siqueira, 2006; Souza et al., 2010).

Os gêneros Acaulospora e Glomus foram os mais abundantes nas áreas estudadas, sendo Acaulospora encontrado em todas as áreas. Em um estudo realizado em áreas de cacau-cabruca localizadas nos municípios de Ilhéus e Aurelino Leal na região Sul da Bahia, Oliveira Filho (2019) também observou predominância desses gêneros nas áreas avaliadas. Os gêneros Acaulospora e Glomus são comuns e adaptados a diferentes características de solos e ecossistemas (Bonfim et al., 2013), têm grande número de espécies conhecidas e os propágulos formados durante a colonização promovem alta infectividade, contribuindo para o sucesso desses táxons (Hart \& Reader, 2002).

Algumas espécies foram exclusivas como Acaulospora sp., Dominikia sp. e Sclerocystis sinuosa registradas apenas na área 1, Glomus glomerulatum na área 2, Gigaspora decipiens, Acaulospora tuberculata, Ambispora appendiculata 
Tabela 5. Espécies de fungos micorrízicos arbusculares (FMAs) em plantios de cacau cabruca na região Sul da Bahia, 2018

\begin{tabular}{lcccccc}
\hline Espécies de FMAs & Área 1 & Área 2 & Área 3 & Área 4 & Área 5 & Área 6 \\
\hline Acaulospora foveata & $\mathrm{X}$ & $\mathrm{X}$ & $\mathrm{X}$ & $\mathrm{X}$ & - & $\mathrm{X}$ \\
Acaulospora longula & - & - & - & - & - & $\mathrm{X}$ \\
Acaulospora mellea & - & - & - & $\mathrm{X}$ & $\mathrm{X}$ & $\mathrm{X}$ \\
Acaulospora morrowiae & $\mathrm{X}$ & $\mathrm{X}$ & $\mathrm{X}$ & - & - & - \\
Acaulospora scrobiculata & $\mathrm{X}$ & $\mathrm{X}$ & $\mathrm{X}$ & $\mathrm{X}$ & - & - \\
Acaulospora sp. & $\mathrm{X}$ & - & - & - & - & - \\
Acaulospora tuberculata & - & - & $\mathrm{X}$ & - & - & - \\
Acaulospora herrerae & - & - & $\mathrm{X}$ & $\mathrm{X}$ & $\mathrm{X}$ & $\mathrm{X}$ \\
Ambispora appendicula & - & - & $\mathrm{X}$ & - & - & - \\
Claroideoglomus etunicatum & - & $\mathrm{X}$ & - & - & $\mathrm{X}$ & - \\
Dominikia sp. & $\mathrm{X}$ & - & - & - & - & - \\
Funneliformis sp. & - & - & - & - & $\mathrm{X}$ & - \\
Gigaspora decipiens & - & - & $\mathrm{X}$ & - & - & - \\
Glomus brohultii & $\mathrm{X}$ & $\mathrm{X}$ & $\mathrm{X}$ & - & - & - \\
Glomus glomerulatum & - & $\mathrm{X}$ & - & - & - & - \\
Glomus sp. & $\mathrm{X}$ & $\mathrm{X}$ & - & $\mathrm{X}$ & - & - \\
Glomus spinuliferum & - & $\mathrm{X}$ & $\mathrm{X}$ & - & - & - \\
Glomus trufemii & - & $\mathrm{X}$ & $\mathrm{X}$ & $\mathrm{X}$ & - & - \\
Paraglomus brasilianum & - & - & $\mathrm{X}$ & - & - & - \\
Paraglomus occultum & $\mathrm{X}$ & $\mathrm{X}$ & - & - & - & - \\
Sclerocystis sinuosa & $\mathrm{X}$ & - & - & - & - & - \\
Septoglomus furcatum & - & - & - & - & $\mathrm{X}$ & - \\
Septoglomus sp. & - & - & - & - & $\mathrm{X}$ & - \\
\hline Riqueza de Espécies & 9 & 10 & 11 & 6 & 6 & 4 \\
\hline & & & & & & \\
\hline
\end{tabular}

Verificou-se correlação significativa positiva entre a densidade de esporos e o teor de fósforo $(\mathrm{P})$ no solo $(\mathrm{r}=0,73)$. A densidade de esporos e os teores de potássio presente no solo apresentou correlação negativa, porém não foi significativa $(\mathrm{r}=-0,67)$. Não houve correlação significativa entre a densidade de esporos e os teores de magnésio $(\mathrm{Mg})$, cálcio $(\mathrm{Ca})$, potássio $(\mathrm{K})$, matéria orgânica $(\mathrm{MO})$ e $\mathrm{pH}$ do solo. Além dos atributos químicos do solo, fatores como aspectos genéticos da planta, condições climáticas, estado nutricional das plantas dentre outros, influenciam na simbiose micorrízica.

A realização deste estudo sobre a ocorrência e diversidade de fungos micorrízicos arbusculares em áreas de cacau-cabruca, possibilita conhecer a condição micorrízica das plantas de cacau, a população fúngica presente no solo e o efeito de características e Paraglomus brasilianum na área 3, Septoglomus sp., Funneliformis sp., Septoglomus furcatum na área 5 e Acaulospora longula na área 6.

Foi verificada a correlação entre densidade de esporos (DE), taxa de colonização micorrízica das raízes $(\mathrm{CM})$ e os atributos químicos do solo (Tabela 6). Observou-se correlação positiva significativa $(r=0,83)$ entre a taxa de colonização micorrízica e o teor de magnésio $(\mathrm{Mg})$ no solo. A taxa de colonização micorrízica correlacionou-se positivamente $(\mathrm{r}=0,97) \mathrm{com}$ o $\mathrm{pH}$ do solo. Segundo Soti et al. (2014), o nível de colonização micorrízica das raízes está relacionado com o $\mathrm{pH}$ do solo, de modo que se verifica maior grau de colonização micorrízica em solos com pH 5,5 a 6,0.

Entretanto, a taxa de colonização apresentou correlação negativa significativa com o teor de potássio $(\mathrm{K})$ do solo $(\mathrm{r}=-0,75)$. Com relação aos teores de matéria orgânica (MO), Ca e P do solo, não houve correlação significativa com a taxa de colonização micorrízica das raízes de cacau. dos solos nas áreas sobre o estabelecimento da simbiose. Estudos futuros são necessários visando avaliar a eficiência de espécies de fungos micorrízicos isolados destas áreas (nativos) e/ou exóticos na produção de mudas de cacau micorrizadas, bem como o estabelecimento de mudas micorrizadas em campo.

Tabela 6. Coeficiente de correlação linear de Pearson entre a taxa de colonização micorrízica (CM), densidade de esporos (DE) e atributos químicos do solo em plantios de cacau cabruca na região Sul da Bahia, 2018

\begin{tabular}{cll}
\hline $\begin{array}{c}\text { Atributos } \\
\text { químicos do solo }\end{array}$ & $\mathbf{C M}$ & $\mathbf{D M}$ \\
\hline $\mathrm{Mg}$ & $0,83^{*}$ & $0,50^{\mathrm{ns}}$ \\
$\mathrm{K}$ & $-0,75^{*}$ & $-0,67^{\mathrm{ns}}$ \\
$\mathrm{P}$ & $-0,20^{\mathrm{ns}}$ & $0,73^{*}$ \\
$\mathrm{Ca}$ & $-0,22^{\mathrm{ns}}$ & $0,40^{\mathrm{ns}}$ \\
$\mathrm{MO}$ & $-0,57^{\mathrm{ns}}$ & $0,33^{\mathrm{ns}}$ \\
$\mathrm{pH}$ & $0,97^{*}$ & $0,11^{\mathrm{ns}}$ \\
\hline
\end{tabular}

* significativo a $5 \%$ de probabilidade pelo teste t. ${ }^{\text {ns }}$ não significativo a $5 \%$ de probabilidade pelo teste $\mathrm{t}$. 


\section{Conclusões}

Nas áreas de cacau-cabruca estudadas:

1. Foram registrados esporos de fungos micorrízicos em todas amostras de solo coletadas;

2. As plantas de cacau apresentavam estruturas fúngicas (esporos, vesículas e arbúsculos) no interior de suas raízes;

3. Foram registradas um total de 23 diferentes espécies de fungos micorrízicos arbusculares, destacando-se os gêneros Glomus e Acaulospora;

4. Houve correlação da densidade de esporos e taxa de colonização micorrízica das raízes com teores de $\mathrm{Ca}, \mathrm{P}$ e pH do solo.

\section{Agradecimentos}

À Universidade Federal do Recôncavo da Bahia (UFRB) e ao Programa de Pós-Graduação em Ciências Agrárias, pela oportunidade de realizar este projeto. À Coordenação de Aperfeiçoamento de Pessoal de Nível Superior (CAPES), pelo apoio financeiro concedido. À Universidade Estadual de Santa Cruz (UESC), por conceder o espaço e materiais para realização das análises laboratoriais. À Universidade Federal do Rio Grande do Norte, onde foi realizada a identificação taxonômica das espécies dos FMAs.

\section{Literatura Citada}

AGGANGAN, N. S.; CORTES, A. D.; REAÑO, C. E. 2019. Growth response of cacao (Theobroma cacao L.) plant as affected by bamboo biochar and arbuscular mycorrhizal fungi in sterilized and unsterilized soil. Biocatalysis and Agricultural Biotechnology 22(1):101-347.

BENJAMIN, C. S. et al. 2016. Cacao families and parents selected as resistant to natural infection of Moniliophthora perniciosa. Crop Breeding and Applied Biotechnology 16(2):141-146.

BONFIM, J. A. et al. 2010. Fungos micorrízicos arbusculares (FMA) e aspectos fisiológicos em cafeeiros cultivados em sistema agroflorestal e a pleno sol. Bragantia 69(1):201-206.

BONFIM, J. A. et al. 2013. Arbuscular mycorrhizal fungi in the Brazilian Atlantic forest: A gradient of environmental restoration. Applied Soil Ecology 71:7-14.

CARRENHO, R. 1998. Influência de diferentes espécies de plantas hospedeiras e fatores edáficos no desenvolvimento de FMA. Tese Doutorado. São Paulo, SP, Universidade Estadual de São Paulo. 135p.

DODD, J. C. 2000. The role of arbuscular mycorrizal fungi in agro - and natural ecosystems. Outlook on Agriculture 29(1):55-62.

EOM, A. H.; HARTNETT, D. C.; WILSON, G. W. T. 2000. Host plant species effects on arbuscular mycorrhizal fungal communities in tallgrass prairie. Oecologia 122:435- 444.

FREW, A. 2019. Arbuscular mycorrhizal fungal diversity increases growth and phosphorus uptake in $\mathrm{C} 3$ and $\mathrm{C} 4$ crop plants. Soil Biology and Biochemistry 135(1):248-250.

GERDEMANN, J. W.; NICOLSON, T. H. 1963. Espores of mycorrhizal Endogone species extracted from soil by wet-sieving and decanting. Transactions of the British Mycological Society 46(1):235-244.

GIOVANNETTI, M.; MOSSE, B. 1980. An evaluation of techniques to measure vesicular-arbuscular mycorrhizal infection in roots. New Phytologist 84(3):484-500.

GROSS, E.; PEREIRA, L. S. 2018. Fungos micorrízicos arbusculares em cacaueiro. In: Souza Júnior, J. O. de. (org.). Cacau - Cultivo, Pesquisa e Inovação. 1ed. Ilhéus, BA, Editus, 018. pp.235-253.

HART, M. M.; READER, R. L. 2002. Taxonomic basis for variation in the colonization strategy of arbuscular mycorrhizal fungi. New Phytologist 153:335-344.

JENKINS, W. R. 1964. A rapid centrifugal - flotation technique for separating nematodes from soil. Plant Diasease Report 48(1):692.

KOSKE, R. E.; GEMMA, J. N. 1989. A modified procedure for staining roots to detect VA mycorrhizas. Mycological Research 92(1):488-505.

LI, C. U. I. et al. 2019. Arbuscular mycorrhizal fungi combined with exogenous calcium improves the growth of peanut (Arachis hypogaea L.) 
seedlings under continuous cropping. Journal of Integrative Agriculture 18(2):407-416.

LIMA, F. S.; SOUSA, C. S. 2014. Crescimento e nutrição de mudas de clones de eucalipto inoculadas com fungos micorrízicos. Pesquisa Agropecuária Tropical (Brasil) 44(2):110-118.

MOREIRA, F. M. S.; SIQUEIRA, J. O. 2006. Microbiologia e bioquímica do solo. Lavras, MG, UFL. 625p.

MORTON, J. B.; BENTIVENGA, S. P.; WHEELER, W. W. 1993. Germ plasm in the International Collection of Arbuscular and Vesicular-Arbuscular Mycorrhizal Fungi (INVAM) and procedures for culture development, documentation and storage. Mycotaxon 48(1):491-528.

OLIVEIRA FILHO, L. J. X. 2019. Caracterização de indicadores da qualidade do solo em sistemas agroflorestais. Dissertação Mestrado. Ilhéus, BA, Universidade Estadual de Santa Cruz. 76p.

PRADO, I. G. O. et al. 2019. Revegetation process increases the diversity of total and arbuscular mycorrhizal fungi in areas affected by the Fundão dam failure in Mariana, Brazil. Applied Soil Ecology 141(1):84-95.

R CORE TEAM. 2019. R: A language and environment for statistical computing. R Foundation for Statistical Computing, Vienna, Austria. URL https:/ /www.R-project.org.

SCHENCK, N. C.; PEREZ, Y. 1988. Manual for the identification of VA mycorrhizal fungi, 2 ed. Gainesville, University of Florida. 241p.

SOTI, P. G. et al. 2014. Mycorrhizal symbiosis and Lygodium microphyllum invasion in south
Florida. A biogeographic comparison. Symbiosis 62(2):81-90.

SOUZA, G. I. A. et al. 2010. Arbuscular Mycorrhizal fungi in agricultural and forest systems. Global Science and Technology 3(2):1-9.

THIOUB, M. et al. 2019. Arbuscular mycorrhizal fungi inoculation enhances phosphorus use efficiency and soybean productivity on a Haplic Acrisol. Soil And Tillage Research 192(1):174-186.

TOLEDO-HERNÁNDEZ, M.; WANGER, T. C.; TSCHARNTKE, T. 2017. Neglected pollinators: Can enhanced pollination services improve cocoa yields? A review. Agriculture, Ecosystems \& Environment 247(1):137-148.

VANHOVE, W.; VANHOUDT, N.; VAN DAMME, P. 2016. Effect of shade tree planting and soil management on rehabilitation success of a 22year-old degraded cocoa (Theobroma cacao L.) plantation. Agriculture, Ecosystems \& Environment 219(1):14-25.

VÁSQUEZ, Z. S. et al. 2019. Biotechnological approaches for cocoa waste management: A review. Waste Management 90(1):72-83.

WANG, F. 2017. Occurrence of arbuscular mycorrhizal fungi in mining-impacted sites and their contribution to ecological restoration: Mechanisms and applications. Critical Reviews in Environmental Science and Technology 47(20):1901-1957.

YANG, Y. et al. 2016. The roles of arbuscular mycorrhizal fungi (AMF) in phytoremediation and tree-herb interactions in $\mathrm{Pb}$ contaminated soil. Scientific Reports 6(1):20469. 